\title{
Radiation-inducible promoter mediated PUMA gene in the the treatment of Tca8113
}

\author{
Dong-Sheng Yu ${ }^{1}$, Wei Zhao ${ }^{1}$, Jia-Xian Luo ${ }^{1}$, Bing Liu ${ }^{1}$, Xiao-Lin Wu ${ }^{2}$ \\ ${ }^{1}$ Institute of Stomatological Research, Sun Yat-sen University, Guangzhou, China; \\ ${ }^{2}$ Department of Stomatology, The Conde S. Januário Hospital Centre, Macao, China. \\ Email: gdyudongsheng@yahoo.com.cn
}

Received 12 August 2011; revised 20 September 2011; accepted 3 October 2011.

\begin{abstract}
Objective: The aim of this study is to explore the therapeutic effect of the PUMA gene mediated by radiation-inducible promoters in the treatment of tongue squamous cell carcinoma. Methods: Recombinant pcDNA3.1 (+)/E-PUMA was constructed, in which the PUMA gene was mediated by a synthetic radiation inducible promoter. The recombined plasmids were transfected into the Tca8113 cell and xenografts of human tongue squamous carcinoma in naked mice respectively. After $24 \mathrm{~h}$, the tumors were treated with $3 \mathrm{~Gy}$ of irradiation to upregulate the PUMA gene expression. PUMA mRNA was detected by RT-PCR. Proliferating cell nuclear antigen (PCNA) and apoptosis were detected by immunohistochemical method and in situ end-labeling (ISEL) respectively. The data were analyzed using the SPSS11.0 software package for chi-square test. Results: Compared with the control group, the comparative survival rate of Tca8113 cells in the PUMA/IR group was markedly decreased and the xenografts were signifycantly suppressed. Up-regulation of PUMA gene expression was observed in the Tca8113 cells and in the xenografts after irradiation. The apoptosis indices of the Tca8113 cells and xenograft with irradiation were markedly higher than those without irradiation. At the same time, the proliferation indices of the Tca8113 cells and xenografts with irradiation were markedly lower than those without irradiation. Conclusions: radiation-inducible promoters can serve as molecular switches to improve the expression of PUMA gene in tongue squamous cell carcinoma both in vivo and in vitro. Low-dose induction radiation can significantly improve therapeutic efficiency.
\end{abstract}

Keywords: Blast; Graph Theory; Redundant Sequences; CD-HIT

\section{INTRODUCTION}

The p53 up-regulated modulator of apoptosis (PUMA) was newly discovered in 2001, which possessed powerful pro-apoptotic effect as a member of the Bcl-2 family and the key protein during the apoptosis caused by radiation [1-3]. Although its specific mechanism for inducing apoptosis needs further explanation, the proapoptotic function has achieved rather good results, becoming a promising new target in gene therapy [4-6]. Radiation-inducible promoters can regulate and control the downstream therapeutic gene expression, a new strategy for radiation-gene therapy founded in recent years $[7,8]$. It combines traditional radiation treatment with gene therapy, which may reduce radiation dosage and increases efficacy of gene therapy $[9,10]$. Human tongue squamous cell carcinoma is the most common type of neoplasm in the oral cavity [11,12]. Little is known about the role of PUMA in the targeted treatment of human tongue squamous cell carcinoma. This research adopted radiation-inducible promoters to mediate PUMA gene for treating human tongue squamous cell carcinoma in vivo or in vitro, to observe the synergistic effect of radiation and to explore a new policy for the targeted therapy of tongue squamous cell carcinoma.

\section{MATERIALS AND METHODS}

\subsection{Cells and Cell Culture}

Tongue squamous carcinoma cells (China Center for Type Culture Collection Wuhan University Hubei China) were cultured in RPMI 1640 (GIBCO, Carlsbad, CA, USA) culture medium supplemented with $10 \%$ fetal calf serum (GIBCO), $50 \mathrm{U} / \mathrm{ml}$ penicillin $\mathrm{G}$, and $50 \mu \mathrm{g} / \mathrm{ml}$ streptomycin. Subculture occurred weekly (seeded at $5 \times$ $10^{5}$ cells $/ \mathrm{ml}$ ), with feeding with complete medium twice a week. All cells were cultured in a humidified 5\% $\mathrm{CO}_{2}$ environment at $37^{\circ} \mathrm{C}$.

\subsection{Animals}

$\mathrm{BALB} / \mathrm{c}$ naked mice were provided by the Experimen- 
tal Animal Center of Sun Yat-sen University. Animal experiments were performed with the permission from the Animal Ethical Commission of Sun Yat-sen University, China.

\section{3. pcDNA (+)-3.1/E-PUMA Construction}

All restriction and modifying enzymes were supplied by Promega or Roche and were used according to the manufacturer's instructions. DNA isolation and purification were carried out using the appropriate kit from Qiagen (Crawley, UK). Nhe I and Hind III were used for conducting double digestion of pMD18-T-E, producing DNA fragments containing radiation inducible promoter sequences. The six-CArG elements enhancer (E6) was cloned using the complementary single-strand-edoligodeoxyribo-nucleotides pair: 5'-AGATCTGC TAGC(CCATATAA-GG) ${ }_{6}{ }^{-}$ TCGCGAA-3' and 5'-T CGCGA(CCTTATATGG) ${ }_{6}$ GCTAGCAGATCTA-3', which were synthesized by the Songon Company, Shanghai, China. Linkers were produced by mixing $0.05 \mathrm{nmol}$ of each oligonucleotide in $5 \mu \mathrm{l}$ volumes, heating them at $55^{\circ} \mathrm{C}$ for $5 \mathrm{~min}$ and then cooling them to room temperature. They were inserted into PUMA upstream of the plasmid pcDNA $(+) 3.1-P U M A, o b-$ taining the eukaryotic expression plasmid pcDNA $(+) 3.1-$ E-PUMA, which carries the radiation inducible promoter mediated PUMA gene.

\subsection{The Killing Effect of PUMA Gene Mediated by Radiation-Inducible Promoter in Vitro}

Grouping. Tca8113 cells were divided into four groups as follows:

Control group: no intervention; PUMA group: PUMA gene transfection only; IR group: irradiation only; PUMA/IR group: PUMA gene transfection and irradiation.

Transfection of DNA. Tca8113 cells were transfected with Lipofectamine (GIBCO) by modifying the manufacturer's procedure based on our previous study $[7,8]$. Tca8113 cells $\left(2 \times 10^{5}\right)$ were seeded into a six-well plate at $2 \times 10^{5}$ cells per well, and conventionally maintained for $24 \mathrm{~h}$. A total of 24 wells of Tca8113 cells were prepared to be transfected. Two micrograms of plasmid DNA $(10 \mu \mathrm{L})$ was mixed with $90 \mu \mathrm{L}$ of serum-free medium. This mixture was then incubated with a mixture of $15 \mu \mathrm{L}$ lipofectamine $(2 \mathrm{mg} / \mathrm{mL})$ and $85 \mu \mathrm{L}$ serum-free medium for $45 \mathrm{~min}$ at room temperature. Subsequently, the DNA-Lipofectamine complex $(200 \mu \mathrm{L})$ was added to each well.

Radiation procedure. After transfection for $24 \mathrm{~h}$, the transfected cells were irradiated by linear accelerator (Primvs, Siemens, Germany) at a dose of 3 Gy. We chose the radiation dose of 3 Gy based on our previous research [8]. The monolayers of the transfected cells in the control group were also mock-irradiated and subse qu- ently treated identically as with the irradiated monolayers of transfected cells.

Microculture tetrazolium assay. A micro-culture tetrazolium (MTT) was used to assess cell viability. All experiments were performed in triplicate. The results were expressed as percentages of the control, which was considered $100 \%$.

RNA preparation and RT-PCR. After IR for $48 \mathrm{~h}$, total mRNA from transfected Tca8113 cells was extracted using an RNeasy Mini Kit (Qiagen, USA) according to the manufacturer's in structions. RT-PCR was carried out using a reverse transcription kit (Promega, USA) and human $\beta$-actin gene was used as the internal control. The PUMA gene primers were as follows: 5'-TGTCGAATAAACGCTTTACAAAC-3' (forward) and 5'AACGTTTGTAATG ATGG CTTCTG-3' (reverse). The $\beta$-actin primers were as follows: 5'-GGTCGGAGTCAACGGATTTGGTCG-3' (forward) and 5'-CCTCCGACGCCTGCTTCAC CAC-3' (reverse). The transcript levels were normalized according to transcription of the $\beta$ actin internal control, and the products were resolved by agarose gel electrophoresis. The intensity was quantified by image-analysis software (NIH Image; Apple, Cupertino, CA, USA).

Detection of apoptosis by flow cytometry. After transfection for $48 \mathrm{~h}$, both adherent and floating cells were harvested and centrifuged. The cell pellets were washed with phosphate-buffered saline (PBS) and then fixed with overnight with $70 \%$ ethanol. After incubation in phosphate-citric acid, the cells were resuspended in PBS containing propidium iodide $(10 \mu \mathrm{g} / \mathrm{mL})$ and RNase (10 $\mu \mathrm{g} / \mathrm{mL})$.The cells were quantified with a FACScan flow cytometer (Becton Dickinson). Salmon sperm DNA (ssDNA) was used as the DNA toxicity control.

\subsection{Tumor Inhibition Effect in Vivo}

Establishment of transplantable tumor model. The freeze-stored Tca8113 cells were revived in an incubator with $5 \% \mathrm{CO}_{2}$ at $37^{\circ} \mathrm{C}$, and subcultured in RPMI-1640 containing $100 \mathrm{~mL} / \mathrm{L}$ fetal bovine serum. The trypsin method was utilized to collect the cancer cells. Naked mice 6 to 8 weeks old were given subcutaneous injecttions at $6 \times 10^{6}$ inoculated tumor cells for each mouse. When the diameters of transplanted tumor reached $8 \mathrm{~mm}$, the treatments were begun.

Plasmid transfection and radiation therapy. The mice with transplanted tumors were randomly divided into four groups with 10 mice for each group: (A) control group, (B) transfection group, (C) radiation group, and (D) transfection and radiation group. Then, $2 \mu \mathrm{g}$ of the plasmid pcDNA3.1 (+)/E-PUMA was dissolved into $100 \mu \mathrm{L}$ of serum-free RPMI-1640 culture medium. At the same time, $80 \mu \mathrm{L}$ serumfree RPMI-1640 culture me- 
dium was used to dilute $20 \mu \mathrm{L}$ Lipofectamine. Both of them were mixed and shaken well, and another $800 \mu \mathrm{L}$ of serum-free RPMI-1640 culture medium was added into the mixture. This prepared miscible liquid was used for intratumoral injection $(100 \mu \mathrm{L} /$ mouse). After $24 \mathrm{~h}$ transfection, groups $\mathrm{C}$ and D were exposed to $3 \mathrm{~Gy}$ of radiation via a linear accelerator.

Description of tumor growth curve. Vernier caliper was used to measure maximum of the tumors and maximum transverse diameter of tumors (b) every four days, and the maximum tumor size of multiple tumors were recorded. Tumor size was calculated using the formula $\mathrm{V}=$ $1 / 2\left(a \times b^{2}\right)$. On the $20^{\text {th }}$ day of transfection, the animals were euthanized and tumor growth curves were constructed.

RNA preparation and RT-PCR. After transfection for $96 \mathrm{~h}$, the naked mice were sacrificed and the tumor tissues were subsequently collected. RT-PCR was employed as in the in vitro experiment described above.

Detect PCNA expression. The proliferating cell nuclear antigen (PCNA) was detected with immunohistochemical kit (Boster, Wuhan, China) according to the manufacturer's protocol: Briefly, the tumor specimens were conventionally fixed, embedded, sectioned, deparaffinized, incubated for $15 \mathrm{~min}$ at $\mathrm{RT}$ in $3 \% \mathrm{H}_{2} \mathrm{O}_{2}$ diluted in methanol; add diluted primary antibody and incubate overnight at $4{ }^{\circ} \mathrm{C}$; The sections were then washed three times with buffer solution, secondary antibodies (rabbit anti-rat monoclonal antibodies 1/75 dilution) were added, and the specimens were incubated for $30 \mathrm{~min}$ at room temperature. $\mathrm{ABC}$ reagent was added and the sections were incubated for $30 \mathrm{~min}$ and immersed in hematoxylin for $90 \mathrm{~s}$ for counterstaining. Primary antibodies were replaced with PBS for the blank control whereas oral mucosal cells were used for the positive control.

Proliferation indices (PIs) were calculated by observing the proportion of positively staining cells. Staining was considered positive when the cell nuclei had been stained brown. The number of positive cells and total observed cells was counted under light microscopy ( $\times 400$ magnification) with a $10 \times 10$ grid. The observers randomly selected distinct fields during three separate evaluations in each slide. Ten sections were used to evaluate every group. PIs were calculated using the following formula: $\mathrm{PI}=$ (positive ly staining cells/total observed cells) $\times 100 \%$.

In situ end-labeling (ISEL) detects apoptosis. Apoptosis was detected by in situ end-labeling (ISEL) with an apoptosis labeling kit (Boster, Wuhan, China) according to the manufacturer's protocol. Tumor specimens were conventionally fixed, embedded, sectioned, and deparaffinized. Endogenous peroxidases were then blocked by immersing the slides in $3 \% \mathrm{H}_{2} \mathrm{O}_{2}$ for $30 \mathrm{~min}$ at room temperature. The slides were incubated in ISEL solution in an $18^{\circ} \mathrm{C}$ ice bath for $2 \mathrm{~h}$. ABC solution was then applied and the slides were incubated at room temperature for $30 \mathrm{~min}$. DAB was subsequently applied for $5 \mathrm{~min}$ at room temperature. Counterstaining was done by immersion in hematoxylin for $60 \mathrm{~s}$. Finally, the slides were rehydrated and topped with coverslips. For the blank control, the DIG-dUTP was replaced with TBS solution, whereas oral mucosal cells were used in the positive control.The calculation of AI is the same as that of PI.

\subsection{Statistical Analysis}

All experiments, as well as the measurements on each experiment, were run in triplicate. The experimental data are presented as means \pm s.d. Statistical analyses were performed by a one-way ANOVA and chi-square test using SPSS software 11.0 (SPSS Inc., Chicago, IL, USA). A $P$ value of 0.05 was considered statistically signifycant.

\section{RESULTS}

\subsection{Inhibition of Tca8113 Growth Cells in Vitro}

The variability of Tca8113 cells was detected by MTT assay. Figure 1 shows that Tca8113 cells grew rapidly in the blank control group. The Tca8113 cells in the PUMA group and the IR group were significantly inhibited compared with the control group. Furthermore, the growth of Tca8113 cells in the PUMA/IR group was significantly more inhibited than in any other group.

\subsection{Expression of PUMA mRNA in Transfected Tca8113 Cells}

After IR for $48 \mathrm{~h}$, the PUMA expression was detected by RT-PCR. Figure 2 shows that the mRNA levels in the PUMA/IR group $(0.782 \pm 0.035)$ were significantly

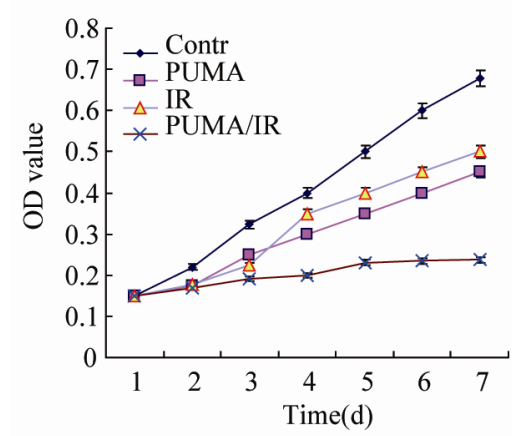

Figure 1. Tca8113 cell growth in vitro. The growth of Tca8113 cells in the PUMA/IR group was significantly inhibited compared with any other group. There was significant difference between the PUMA group and the PUMA/IR group. 


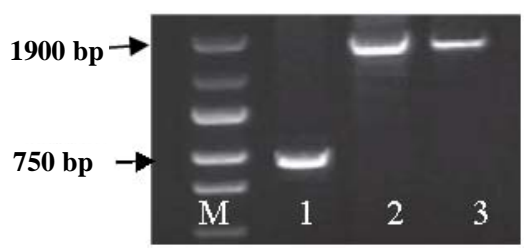

Figure 2. PUMA transcription after transfection into Tca8113 cells. Lines M, DNA maker; Line 1, product of $\beta$-actin (750 $\mathrm{bp)}$; Line 2, product of PUMA gene in the PUMA/IR group; Line 3, product of PUMA gene in the PUMA group.

higher than those in the PUMA group $(0.527 \pm 0.013$, P $<0.01)$. This result suggests that the synthetic radiation inducible promoters combined with IR up-regulated PUMA gene expression in the transfected Tca8113 cells.

\subsection{FACScan Analysis of Cells Apoptosis}

After IR for $48 \mathrm{~h}$, the percentage of cell apoptosis in the PUMA/IR group was about $(26.32 \pm 2.32) \%$, which is significantly higher than the $(16.65 \pm 1.44) \%$ in the PUMA group $(\mathrm{P}<0.05)$ and the $(9.76 \pm 0.85) \%$ in the IR group $(\mathrm{P}<0.01)$ (Figure 3). These results indicate that IR marked enhanced the anticancer ability of PUMA gene therapy in vitro.

\subsection{Tumor Growth Curve}

The transplanted tumor growth curves of the naked mice from different groups are shown in Figure 4. PUMA gene transfection and 3Gy irradiation exposure have certain inhibitory effects on tumor growth, but are obviously lower than that of the radiation gene therapy.

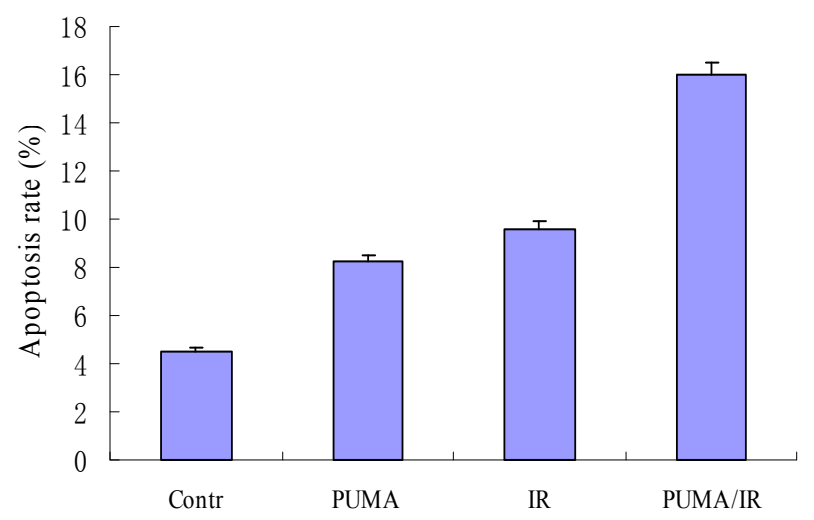

Figure 3. Cells apoptosis in different group were analyzed by flow cytometry $48 \mathrm{~h}$ after irradiation. The apoptosis index was highest $(26.32 \pm 2.32) \%$ in the PUMAMR group, which was significantly higher than $(16.65 \pm 1.44)$ in the PUMA group (P $<0.05)$ and $(9.76 \pm 0.85) \%$ in the IR group $(\mathrm{P}<0.01)$.

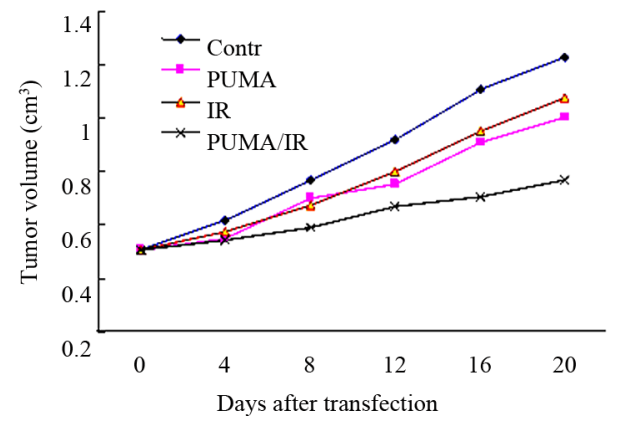

Figure 4. The growth curves of tumors in naked mice. Tumor growth was fast in the blank control group, but significantly depressed in the IR, PUMA and PUMAMR groups. Furthermore, there was a significant difference between the PUMA group and PUMA/IR group.

\subsection{PUMA mRNA Expression in the Xenografts}

PUMA gene mRNA expression is merely detected in the control group and the IR group. The PUMA gene fragment was detected in both the PUMA group and the PUMA/IR group (Figure 5), but the band intensity of the former is significantly higher than that of the latter. Computer image analyzer scans of the electrophoretic band grey values were compared with the $\beta$-actin grey value and their ratios are shown in Table 1. The differences between the two groups are significant $(P<0.05)$. Radiation treatment increases PUMA expression level.

\subsection{PCNA Staining and ISEL Labeling Results}

The PCNA staining and ISEL labeling results are shown in Figures 6(a)-(d) and Figure 6(A)-(D), whereas the PI and AI results are in Table 2.

\section{DISCUSSION}

Apoptosis is a gene-determined active cell death. It plays a key role in the normal development of multi-cellular organisms. The occurrence of tumor is closely linked with

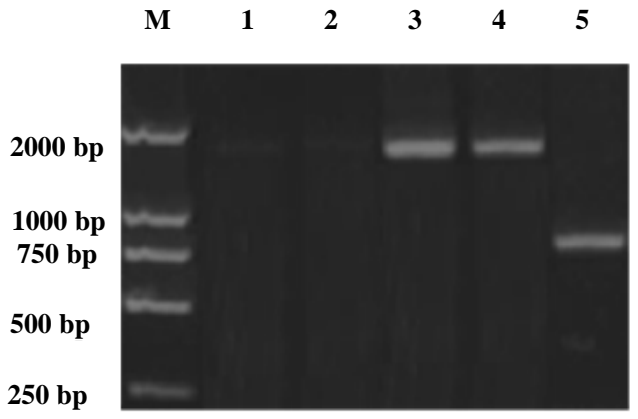

Figure 5. Agarose gel electrophoresis of RTPCR products M: DNA marker; 1: control group; 2: radiation group; 3: tranfection and radiation group; 4: tranfection group. 
Table 1. Comparison of grey level of agarose gel electrophoresis.

\begin{tabular}{cc}
\hline Group & Mean $(\chi \pm \mathrm{s})$ \\
\hline PUMA group & $0.826 \pm 0.081$ \\
PUMA/IR group & $1.031 \pm 0.096$ \\
\hline
\end{tabular}

Table 2. The AIs and PIs in the different groups $(\chi \pm \mathrm{s} \%)$.

\begin{tabular}{ccc}
\hline Group & PI (\%) & AI (\%) \\
\hline control & $43.54 \pm 6.42$ & $8.76 \pm 1.51$ \\
IR & $35.26 \pm 5.31^{\mathrm{a}}$ & $12.33 \pm 3.11^{\mathrm{a}}$ \\
PUMA & $33.44 \pm 4.32^{\mathrm{a}}$ & $4.76 \pm 4.12^{\mathrm{a}}$ \\
PUMA/IR & $25.68 \pm 5.43^{\mathrm{ab}}$ & $28.98 \pm 5.22^{\mathrm{ab}}$ \\
\hline
\end{tabular}

${ }^{\mathrm{a}}$ Compared with control, $\mathrm{P}<0.05 ;{ }^{\mathrm{b}}$ Compared with PUMA, $\mathrm{P}<0.05$.

abnormal apoptosis [13-15]. Although many p53-regulated genes participate in apoptosis, PUMA is currently considered one of the key genes in the process $[1,2,16]$. PUMA belongs to the BH-3 subfamily of the bcl-2 protein family. The BH-3 structural domain and its position in the mitochondria are indispensable in the induction of apoptosis and inhibition of cell growth [1,17]. PUMA induces apoptosis through p53-dependent and non-p53 dependent pathways. Differing from other target spots, PUMA not only regulates expression through common p53 responsive elements located in its promoter, but also induces expression by non-p53 dependent mechanisms, such as glucocorticoids, removal of growth factors, ki- nase inhibitors and phorbol esters $[18,19]$. While studying the mechanism of myoblast apoptosis during the differentiation and development process of the skeletal muscle, Shaltouki et al. [20] found that PUMA can directly mediate the release of cytochrome $\mathrm{C}$ through a non-p53dependent pathway, thereby activating Caspase- 9 and causing myoblast apoptosis. The function of PUMA is unaffected by p53 expression. In experiments by Wang [21], there was no correlation between p53 expression and the inhibition of proliferation and the induction of apoptosis in esophageal cancer cell lines, including KYSE-150, KYSE-410, KY SE-510, and YES-2. Exogenous transfection of PUMA into human melanoma cell systems causes apoptosis regardless of p53 expression. Hideaki reported that extensive apoptosis occurred in the expression mutation and wild characteristic of four PUMA-transfected cell lines [22]. Wang also found that the inhibition of proliferation and induction of apoptosis by Ad-PUMA in esophageal cancer cell lines were better than that of Ad-p53 [21]. Apoptosis induction by PUMA is independent of $\mathrm{p} 53$ expression, and its effect is better than that of $\mathrm{p} 53$, which makes it a potential target for gene therapy.

PUMA is a key protein in apoptosis, and radiation damage can result in PUMA activation, leading to apoptosis through a series of complex mechanisms [23] Although its specific induction mechanism requires further explanation, the application of the pro-apoptotic function of p53 in anti-tumor experiments has achieved fairly
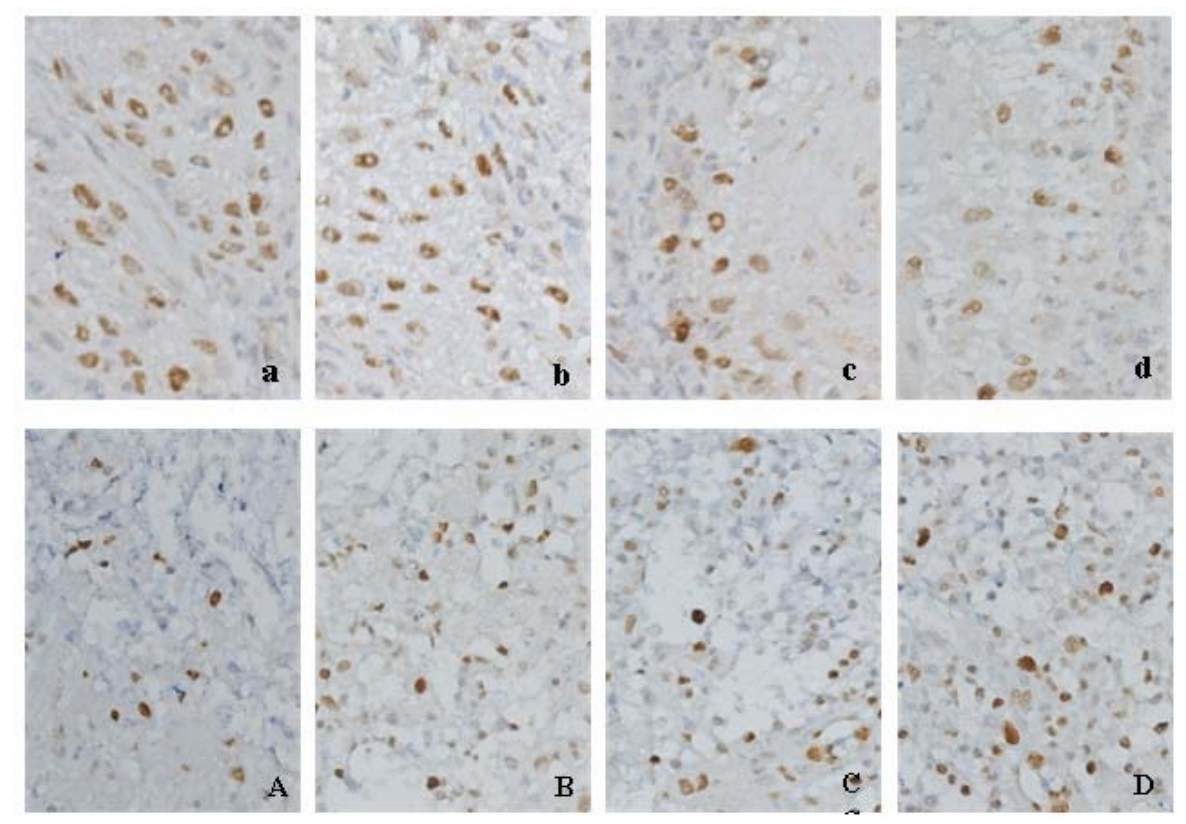

Figure 6. Expression of PCNA and apoptosis cells in different group tumors. Expression of PCNA (SABC, $\times 400$ ) in the: (a) control group; (b) IR group; (c) PUMA group; (d) PUMA/IR group. Apoptotic cells (ISEL, $\times 400$ ) in the: (A) control group; (B) IR radiation group; (C) PUMA group; (D) PUMA/IR group. 
good results. Yu et al. found that PUMA gene transfection could enhance the sensitivity of lung cancer cells to radiotherapy [24].

There may be serious side effects in patients receiving radiotherapy. Decreasing the occurrence of these side effects will be beneficial for cancer patients. PUMA gene transfection can promote the apoptosis of tumor cells and enhance its sensitivity to radiotherapy $[25,26]$. Currently, methods to increase PUMA-targeted expression in tumors are the largest obstacle in the application of PUMA in clinical practice. A new radiation-gene strategy that uses radiation inducible promoters that stimulate downstream gene expression to construct control and regulation systems for therapeutic gene expression has been adopted in the current research [7,27,28]. This system is a typical exogenous regulation mechanism that can selectively kill tumor cells while protecting normal structures from damage [7,29]. With the development of radiotherapy techniques such as intensity modulation radiated therapy and three-dimensional conformal radio therapy, the radiation range can be adjusted to the target areas by regulating the output dosage of various areas in the radiation field [30,31].

To educe synergistic effects by integrating various therapeutic strategies together is a trend in current gene therapy [31,32]. In the current study, we demonstrated that PUMA gene expression in tongue squamous cell carcinoma was significantly upregulated at the transcriptional level by treatment with radiation-inducible promoters following 3 Gy of IR. We also proved that the combination of PUMA gene therapy system with 3 Gy of IR could significantly inhibit the growth of tumor cells by promoting apoptosis and reducing proliferation in vivo and in vitro. Although these results are not yet applicable for the treatment of humans, they indicate that radiation inducible promoters can serve as molecular switches for regulating the expression of the PUMA gene and that low-dose radiation can significantly improve therapeutic targeting efficiency in treatment of tongue squamous cell carcinoma.

\section{ACKNOWLEDGEMENTS}

This study was supported by the National Natural Sciences Foundation of China (Grant No. 3097 3340), the Guangdong Sciences and Technology Project (Grant No. 2008B030301113 and 2011B050400030), and the Guangdong Natural Sciences Foundation (Grant No. 9151008901000187 and S2011020003247).

\section{REFERENCES}

[1] Nakano, K. and Vousden, K.H. (2001) PUMA, a novel proapoptotic gene, is induced by p53. Molecular Cell, 7, 683-694. doi:10.1016/S1097-2765(01)00214-3
[2] Labi, V. and Villunger, A. (2010) PUMA-mediated tumor suppression: A tale of two stories. Cell Cycle, $\mathbf{9}$, 4269-4275. doi:10.4161/cc.9.21.13666

[3] Avila, J.L., Grundmann, O., Burd, R., et al. (2009) Radiation-induced salivary gland dysfunction results from p53-dependent apoptosis. International Journal of Radiation Oncology, Biology, Physics, 2, 523-529. doi:10.1016/j.ijrobp.2008.09.036

[4] Chen, Y., Xiang, Y. and Lin, C. (2007) PUMA-A promising tumor gene therapy target. Oncology Progress, 5, 282-285.

[5] Michalak, E.M., Vandenberg, C.J., Delbridg, A.R., et al., (2010) Apoptosis-promoted tumorigenesis: Gammairradiation-induced thymic lymph omagenesis requires. Puma-driven leukocyte death Genes Dev, 24, 16081613. doi:10.1101/gad.1940110

[6] Shao, L., Sun, Y., Zhang, Z., et al. (2010) Deletion of proapoptotic PUMA selectively protects hematopoietic stem and progenitor cells against high-dose radiation. Blood, 23, 4707-4714. doi:10.1182/blood-2009-10-248872

[7] Yu, D.-S., Zhao, W., Huang, H.-Z., et al. (2010) Synthetic radiation-inducible promoters mediated HSVTK/GCV gene therapy in the treatment of oral squamous cell carcinoma. Oral Diseases, 16, 445-452. doi:10.1111/j.1601-0825.2010.01655.x

[8] Rao, G.V., Kumar, N.A., Gupta, R., et al. (2010) Radiation-inducible silencing of UPA and UPAR in vitro and in vivo in meningioma. International Journal of Oncology, 4, 809-816.

[9] Nandi, S., Ulasov, I.V., Tyler, M.A., et al. (2008) Low-dose radiation enhances surviving-mediated virotherapy against malignant glioma stem cells. Cancer Research, 14, 5778-5784. doi:10.1158/0008-5472.CAN-07-6441

[10] Soudry, E., Preis, M., Hod, R., et al. (2010) Squamous cell carcinoma of the oral tongue in patients younger than 30 years: Clinicopathologic features and outcome. Clinical Otolaryngology, 4, 307-312. doi:10.1111/j.1749-4486.2010.02164.x

[11] Zhang, T.H., Liu, H.C., Zhu, L.J., et al. (2011) Activation of notch signaling in human tongue carcinoma. Journal of Oral Pathology \& Medicine, 1, 37-45. doi:10.1111/j.1600-0714.2010.00931.x

[12] Elias, S.T., Diniz, J., Almeida, R.S., et al. (2010) Cytotoxic effect of tobacco extracts on human oral squamous cell carcinoma cell-line. Oral Oncology, 12, 869-873. doi:10.1016/j.oraloncology.2010.09.008

[13] Hajji, N. and Joseph, B. (2010) Epigenetic regulation of cell life and death decisions and deregulation in cancer. Eessays in Biochemistry, 1, 121-146. doi:10.1042/bse0480121

[14] Kerr, J.F., Wyllie, A.H. and Currie, A.R. (1972) Apoptosis: A basic biological phenomenon with wideranging implications in tissue kinetics. British Journal of Cancer, 4, 239-257. doi:10.1038/bjc. 1972.33

[15] Qiu, W., Leibowitz, B., Zhang, L., et al. (2010) Growth factors protect intestinal stem cells from radiation-induced apoptosis by suppressing PUMA through the $\mathrm{PI} 3 \mathrm{~K} / \mathrm{AKT} / \mathrm{p} 53$ axis. Oncogene, 11, 1622-1632. doi:10.1038/onc.2009.451 
[16] Chipuk, J.E. and Green, D.R. (2009) PUMA cooperates with direct activator proteins to promote mitochondrial outer membrane permeabilization and apoptosis. Cell Cycle, 17, 2692-2696. doi:10.4161/cc.8.17.9412

[17] Yu, J. and Zhang, L. (2008) PUMA, a potent killer with or without p53. Oncogene, 27, S71-S83. doi:10.1038/onc.2009.45

[18] Hong, L.Z., Zhao, X.Y. and Zhang, H.L. (2010) p53-mediated neuronal cell death in ischemic brain injury. Neuroscience Bulletin, 3, 232-240. doi:10.1007/s12264-010-1111-0

[19] Shaltouki, A., Freer, M., Mei, Y., et al. (2007) Increased expression of the pro-apoptotic $\mathrm{Bcl} 2$ family member PUMA is required for mitochondrial release of cytochrome $\mathrm{C}$ and the apoptosis associated with skeletal myoblast differentiation. Apoptosis, 12, 2143 2154. doi:10.1007/s10495-007-0135-Z

[20] Wang, H., Qian, H., Yu, J., et al. (2006) Administration of PUMAadenovirus increases the sensitivity of esophageal cancer cellsto anticancer drugs. Cancer Biology \& Therapy, 4, 380-389. doi:10.4161/cbt.5.4.2477

[21] Hideaki, I., Takao, K. and Toru, M. (2005) Efficacy of PUMA for malignant glioma cells regard less of the p53 status. Human Gene Therapy, 6, 685-692.

[22] Vousden, K.H. (2005) Apotosis. p53 and PUMA: A deadly duo. Science, 5741, 1685-1686. doi:10.1126/science.1118232

[23] Yu, J., Yue, W., Wu, B., et al. (2006) PUMA sensitizes lung cancer cells to chemotherapeutic agents and irradiation. Clinical Cancer Research, 9, 2928-2935. doi:10.1158/1078-0432.CCR-05-2429

[24] Qiu, W., Carson-Walter, E.B., Liu, H., et al. (2008) PUMA regulates intestinal progenitor cell radio-sen- sitivity and gastrointestinal syndrome. Cell Stem Cell, 6, 576-583. doi:10.1016/j.stem.2008.03.009

[25] Sasano, N., Enomoto, A., Hosoi, Y., et al. (2010) Edaravone, a known free radical scavenger, enhances X-ray-induced apoptosis at low concentrations. Cancer Letters, 1, 52-57. doi:10.1016/j.canlet.2009.12.020

[26] Tsurushima, H., Yuan, X., Dillehay, L.E., et al. (2008) Radiation-inducible caspase-8 gene therapy for malignant brain tumors. International Journal of Radiation Oncology, Biology, Physics, 2, 517-525. doi:10.1016/j.ijrobp.2008.02.002

[27] Marignol, L., Coffey, M., Hollywood, D., et al. (2007) Radiation to control transgene expression in tumors. Cancer Biology \& Therapy, 7, 1005-1012. doi:10.4161/cbt.6.7.4477

[28] Vilaboa, N. and Voellmy, R. (2006) Regulatable gene expression systems for gene therapy. Current Gene Therapy, 4, 421-38. doi: $10.2174 / 156652306777934829$

[29] Bhide, S.A. and Nutting, C.M. (2010) Recent advances in radiotherapy. BMC Medicine, 8, 25-28. doi:10.1186/1741-7015-8-25

[30] Brahme, A. and Lind, B.K. (2010) A systems biology approach to radiation therapy optimization. Radiation and Environmental Biophysics, 2, 111-124. doi:10.1007/s00411-010-0268-2

[31] John-Aryankalayil, M., Palayoor, S.T., Cerna, D., et al. (2010) Fractionated radiation therapy can induce a molecular profile for therapeutic targeting. Radiation Research, 4, 446-458. doi:10.1667/RR2105.1

[32] Marignol, L., Robson, T., McCarthy, H.O., et al. (2008) The tissue plasminogen activator gene promoter: A novel tool for radiogenic gene therapy of the prostate? Journal of Gene Medicine, 9, 1032-1038. doi:10.1002/jgm. 1221 